\title{
Open charm production at high energies and the quark Reggeization hypothesis
}

\author{
B. A. Kniehl* and A. V. Shipilova ${ }^{\dagger}$ \\ II. Institut für Theoretische Physik, Universität Hamburg, Luruper Chaussee 149, 22761 Hamburg, Germany \\ V. A. Saleev \\ Samara State University, Academic Pavlov Street 1, 443011 Samara, Russia
}

(Received 17 December 2008; published 9 February 2009)

\begin{abstract}
We study open charm production at high energies in the framework of the quasi-multi-Reggekinematics approach applying the quark-Reggeization hypothesis implemented with Reggeon-Reggeonparticle and Reggeon-particle-particle effective vertices. Adopting the Watt-Martin-Ryskin unintegrated quark and gluon distribution functions of the proton and photon, we thus nicely describe the proton structure function $F_{2, c}$ measured at DESY HERA as well as the transverse-momentum distributions of $D$ mesons created by photoproduction at HERA and by hadroproduction at the Fermilab Tevatron.
\end{abstract}

DOI: 10.1103/PhysRevD.79.034007

\section{INTRODUCTION}

The study of open charm production in high-energy lepton-hadron and hadron-hadron collisions is considered as a test of the general applicability of perturbative quantum chromodynamics (QCD) and also provides information on the parton distribution functions (PDFs) of protons and photons. The present analysis is to explore our potential to access a new dynamical regime, namely, the highenergy Regge limit, which is characterized by the condition $\sqrt{S} \gg \mu \gg \Lambda_{\mathrm{QCD}}$, where $\sqrt{S}$ is the total collision energy in the center-of-mass (CM) reference frame, $\Lambda_{\mathrm{QCD}}$ is the asymptotic scale parameter of QCD, and $\mu$ is the typical energy scale of the hard interaction. In the processes of heavy-quark production, one has $\mu \geq m$, where $m$ is the heavy-quark mass. In this high-energy regime, the contribution from partonic subprocesses involving $t$-channel parton (quark or gluon) exchanges to the production cross section can become dominant. Thus, the transverse momenta of the incoming partons and their off-shell properties can no longer be neglected, and we deal with Reggeized $t$-channel partons.

The quasi-multi-Regge-kinematics (QMRK) approach $[1,2]$ is particularly appropriate for this kind of high-energy phenomenology. It is based on an effective quantum field theory implemented with the non-Abelian gauge-invariant action, as suggested a few years ago [3]. Our previous analyses of charmonium and bottomonium production at the Fermilab Tevatron [4] demonstrated the advantages of the high-energy factorization scheme over the collinear parton model as far as the description of experimental data is concerned. These observations were substantiated for $B$-meson production at the Tevatron in Ref. [5], where the experimental data were again well described using the

\footnotetext{
*kniehl@desy.de

†alexshipilova@ssu.samara.ru

*saleev@ssu.samara.ru
}

PACS numbers: $12.39 . \mathrm{St}, 12.40 . \mathrm{Nn}, 13.60 . \mathrm{Hb}, 13.85 . \mathrm{Ni}$

Fadin-Lipatov effective Reggeon-Reggeon-gluon vertex [2]. In Ref. [6], where the effective photon-Reggeon-quark vertex was obtained for the first time, the hypothesis of quark Reggeization was successfully used to describe experimental data on single prompt-photon production and on the proton structure functions $F_{2}$ and $F_{L}$.

The CDF Collaboration measured the differential cross sections $d \sigma / d p_{T}$ for the inclusive production of $D^{0}, D^{ \pm}$, $D^{\star \pm}$, and $D_{s}^{ \pm}$mesons in $p \bar{p}$ collisions in run II at the Tevatron as functions of transverse momentum $\left(p_{T}=\right.$ $\left.\left|\vec{p}_{T}\right|\right)$ in the central rapidity $(y)$ region [7]. These measurements were compared with theoretical predictions obtained at next-to-leading order (NLO) in the collinear parton model of QCD [8,9] taking into account quark and hadron mass effects, and it was found that the latter improve the description of the data.

The differential cross sections $d \sigma / d p_{T}$ and $d \sigma / d y$ for inclusive $D^{* \pm}$ and $D_{s}^{ \pm}$photoproduction measured by the H1 [10] and ZEUS [11,12] collaborations at the DESY HERA Collider were compared with NLO predictions in the collinear parton model. For $D^{* \pm}$ mesons, this was done in three approaches: the zero-mass variable-flavor-number scheme (ZM-VFNS) [13,14], the fixed-flavor-number scheme (FFNS) [15], and the general-mass variable-flavor-number scheme (GM-VFNS) [16]. The experimental results were found to generally lie above the NLO expectations. For $D_{s}^{ \pm}$mesons, the calculations were performed in the FFNS [15] and in the model suggested by Berezhnoy, Kiselev, and Likhoded [17].

In this paper, we study $D$-meson production under HERA and Tevatron experimental conditions as well as the charm structure function $F_{2, c}$ of the proton for the first time in the framework of the QMRK approach [1,2] complemented with the quark-Reggeization hypothesis. This paper is organized as follows. In Sec. II, we present the basic formalism of our calculations and briefly recall the QMRK approach in connection with the quarkReggeization hypothesis. In Sec. III, we consider the 
charm structure function $F_{2, c}$ of the proton and compare our results with experimental data. In Secs. IV and V, we describe $D$-meson production via charm-quark fragmentation at HERA and the Tevatron, respectively. In Sec. VI, we summarize our conclusions.

\section{BASIC FORMALISM}

In the phenomenology of the strong interactions at high energies, it is necessary to describe the QCD evolution of the PDFs of the colliding particles (hadrons or photons) starting from some scale $\mu_{0}$ which controls a nonperturbative regime up to the typical scale $\mu$ of the hard-scattering processes, which is typically of the order of the transverse mass $M_{T}=\sqrt{M^{2}+p_{T}^{2}}$ of the produced particle (or hadron jet) with (invariant) mass $M$ and transverse momentum $\vec{p}_{T}$. In the region of very high energies, which corresponds to the so-called Regge limit, the typical ratio $x=\mu / \sqrt{S}$ becomes very small, $x \ll 1$. This leads to large logarithmic contributions of the type $\left[\alpha_{s} \ln (1 / x)\right]^{n}$, where $\alpha_{s}$ is the strong-coupling constant, which are conveniently resummed in the Balitsky-Fadin-Kuraev-Lipatov [18] formalism by the evolution of unintegrated gluon and quark distribution functions $\Phi_{g, q}^{p, \gamma}\left(x, q_{T}^{2}, \mu^{2}\right)$, where $x$ and $\vec{q}_{T}$ are the longitudinal-momentum fraction and transverse momentum of the Reggeized parton with respect to the parent particle, respectively. Correspondingly, in the QMRK approach $[1,2]$, the initial-state $t$-channel gluons and quarks are considered as Reggeons, or Reggeized gluons $(R)$ and quarks $(Q)$. They carry finite transverse momenta $\vec{q}_{T}$ with respect to the hadron or photon beam from which they stem and are off mass shell.

The advantages of the QMRK approach in comparison with the conventional $k_{T}$-factorization scheme [19] include: first, it uses gauge-invariant amplitudes and is based on a factorization hypothesis that is proven in the leading logarithmic approximation; second, it carries over to nonleading orders in the strong-coupling constant, as recently proven [20]. The Reggeization of amplitudes provides the opportunity to efficiently take into account large radiative corrections to processes in the Regge limit beyond what is included in the collinear approximation, which is of great practical importance.

Recently, the Feynman rules for the induced and some important effective vertices of the effective theory based on the non-Abelian gauge-invariant action [3] have been derived in Ref. [21]. However, these rules only refer to processes with Reggeized gluons in the initial state. As for $t$-channel quark-exchange processes, such rules are still unknown, so that it is necessary to construct effective vertices involving Reggeized quarks using QMRK approach prescriptions in each application from first principles. Of course, a certain set of Reggeon-Reggeonparticle effective vertices are known, for example, for the transitions $R R \rightarrow g$ [22], $Q \bar{Q} \rightarrow g$ [23], and $R Q \rightarrow q$ [24].
The effective $\gamma^{*} Q \rightarrow q$ vertex, which describes the production of a quark in the collision of a virtual photon with a Reggeized quark, has been recently obtained in Ref. [6].

In our numerical calculations below, we adopt the prescription proposed by Kimber, Martin, Ryskin, and Watt [25] to obtain unintegrated gluon and quark distribution functions for the proton from the conventional integrated ones, as implemented in Watt's code [26]. To obtain the analogous unintegrated functions for the photon, we modify Watt's code [26]. As input for this procedure, we use the Martin-Roberts-Stirlin-Thorne [27] proton and the GlückReya-Vogt [28] photon PDFs.

\section{CHARM STRUCTURE FUNCTION $F_{2, c}$ OF THE PROTON}

On the experimental side, the charm structure function $F_{2, c}$ of the proton was measured by H1 [29] and ZEUS [30] in deep inelastic scattering (DIS) of electrons and positrons on protons at HERA. In this section, we consider this quantity in the framework of the QMRK approach endowed with the quark-Reggeization hypothesis. We thus need the partonic cross section for the production of a charm quark in the collision of a virtual photon and a Reggeized charm quark. The corresponding Feynman diagram is depicted in Fig. 1(a). The relevant vertex was found in Ref. [6] and reads:

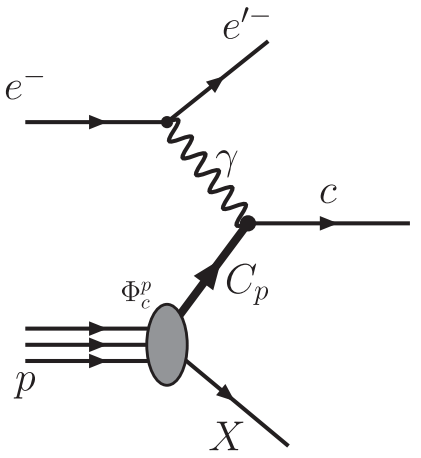

(a)
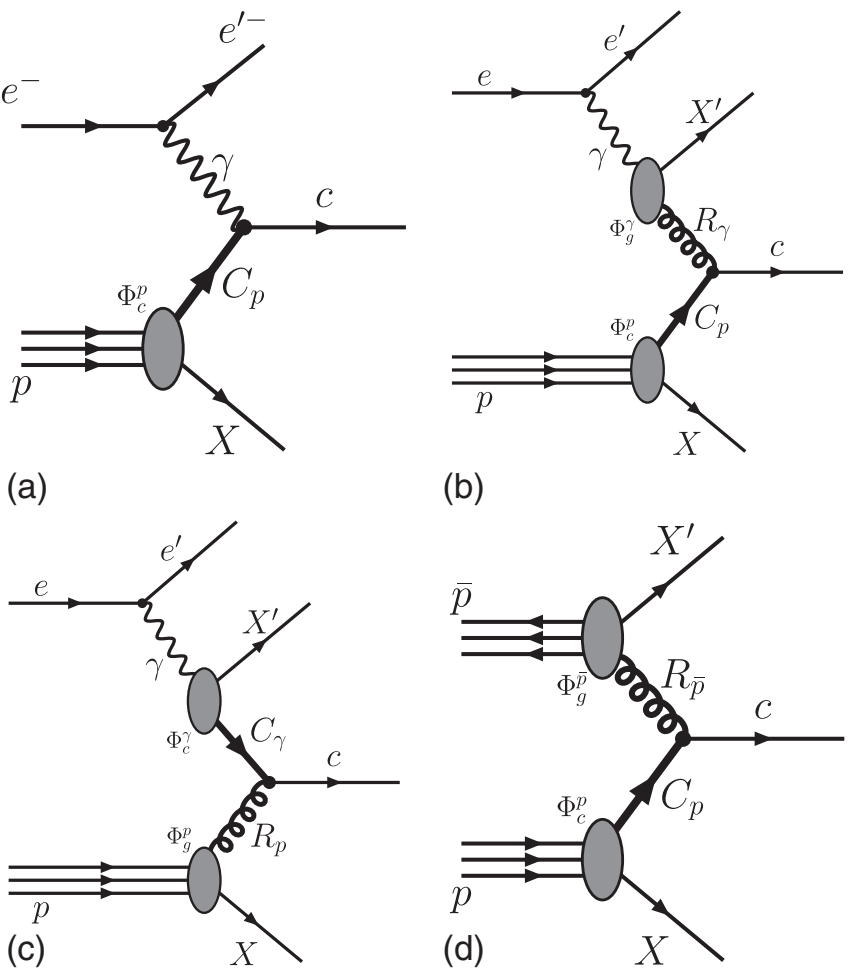

(b)

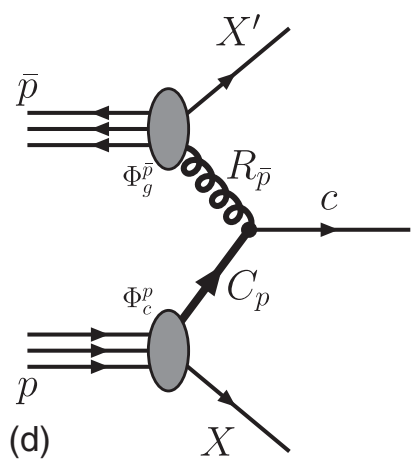

FIG. 1. Feynman diagrams contributing at LO in the QMRK approach with the quark-Reggeization hypothesis to (a) $F_{2, c}$ and direct photoproduction, (b) resolved photoproduction with charm in the proton remnant, (c) resolved photoproduction with charm in the photon remnant, and (d) hadroproduction. 

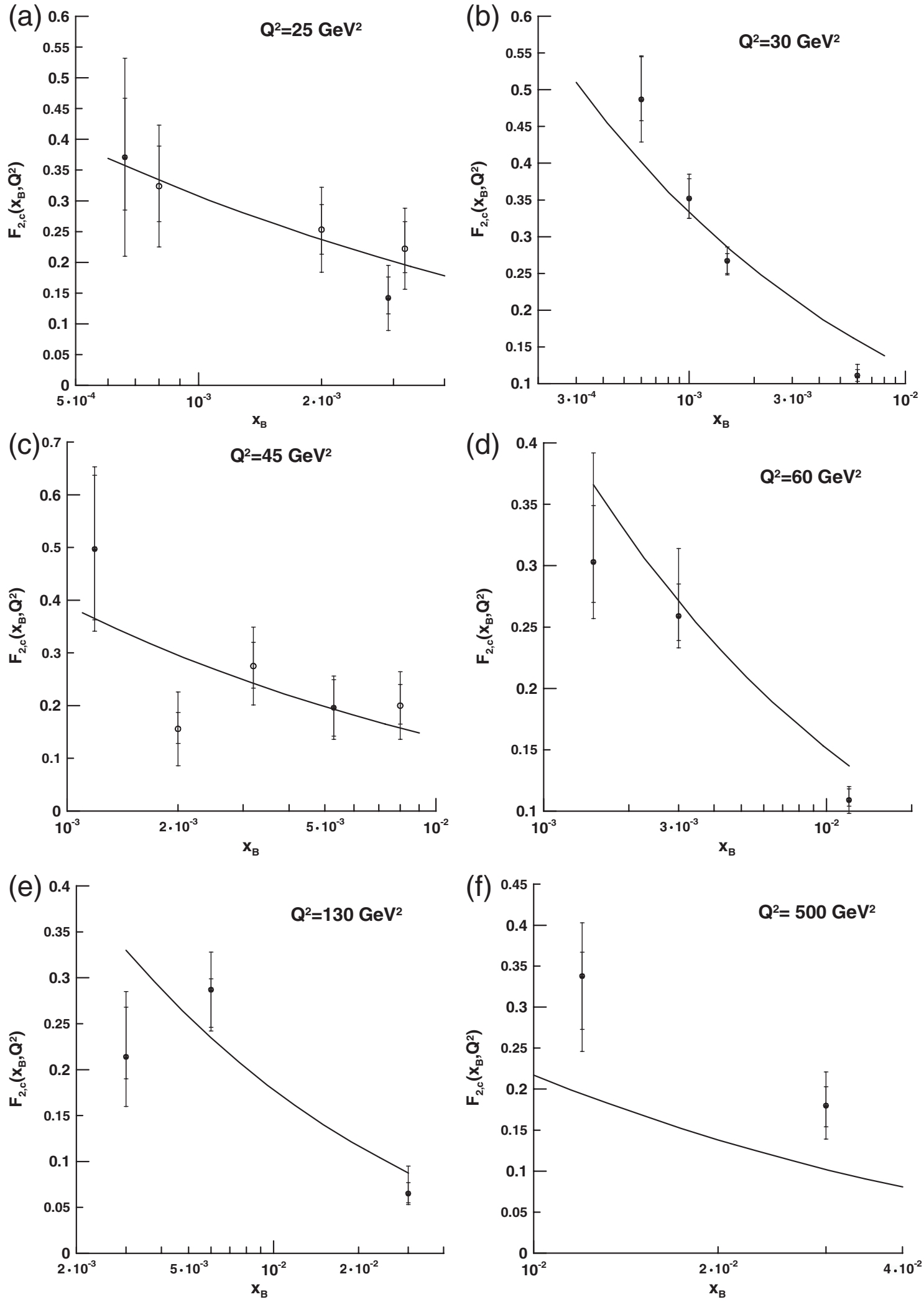

FIG. 2. $\quad F_{2, c}\left(x_{B}, Q^{2}\right)$ as a function of $x_{B}$ at (a) $Q^{2}=25$, (b) 30, (c) 45, (d) 60, (e) 130, and (f) $500 \mathrm{GeV}^{2}$. The H1 [29] (open circles) and ZEUS [30] (filled circles) data are compared with LO predictions from the QMRK approach with the quark-Reggeization hypothesis. 


$$
\begin{aligned}
C_{\gamma Q}^{q}= & -e e_{q}\left[\frac{q_{1}^{2}}{q_{1}^{2}+q_{2}^{2}} \gamma^{\mu}-\frac{2 k^{\mu}}{q_{1}^{2}+q_{2}^{2}} \phi_{2}\right. \\
& \left.+\frac{2 x_{2} q_{2}^{2} P_{2}^{\mu}}{\left(q_{1}^{2}+q_{2}^{2}\right)^{2}} q_{2}\right],
\end{aligned}
$$

where the four-momenta of the virtual photon, the proton, the Reggeized charm quark, and the outgoing charm quark are denoted as $q_{1}, P_{2}, q_{2}=x_{2} P_{2}+q_{2 T}$, and $k=q_{1}+q_{2}$, respectively. We concentrate on photons with large virtuality $Q^{2}=-q_{1}^{2} \gg m_{c}^{2}$, so that the massless approximation for describing DIS structure functions is appropriate [6]. We then obtain the following master formula for $F_{2, c}$ :

$$
\begin{aligned}
F_{2, c}\left(x_{B}, Q^{2}\right)= & 2 e_{c}^{2} \int_{0}^{Q^{2}} d t_{2} \Phi_{c}^{p}\left(x_{2}, t_{2}, \mu^{2}\right) \\
& \times \frac{Q^{2}\left(Q^{4}+6 Q^{2} t_{2}+2 t_{2}^{2}\right)}{\left(Q^{2}+t_{2}\right)^{3}},
\end{aligned}
$$

where $e_{c}=2 / 3$ is the fractional electric charge of the charm quark and $x_{2}=x_{B}\left(Q^{2}+t_{2}\right) / Q^{2}$, with $x_{B}$ being the Bjorken variable. For definiteness, we choose the factorization scale to be $\mu^{2}=Q^{2}$.

In Fig. 2, we compare the $x_{B}$ distributions of $F_{2, c}$ for various values of $Q^{2}$ with the H1 [29] and ZEUS [30] data. We find good agreement for all values of $Q^{2}$, except for the highest one, $Q^{2}=500 \mathrm{GeV}^{2}$, where our prediction is about $50 \%$ below the data. This disagreement shows the importance of higher-order corrections at large values of $Q^{2}$, which are beyond the scope of our present study. For an improved description of $F_{2, c}$ at low values of $x_{B}$ in the collinear parton model, we refer to Ref. [31].

\section{D-MESON PRODUCTION AT HERA}

On the experimental side, ZEUS measured the $p_{T}$ distributions of $D^{* \pm}$ [11] and $D_{s}^{ \pm}$[12] mesons with rapidity [32] $|y| \leq 1.5$ inclusively produced in photoproduction at HERA I, with proton energy $E_{p}=820 \mathrm{GeV}$ and lepton energy $E_{e}=27.5 \mathrm{GeV}$ in the laboratory frame, in the ranges $2 \leq p_{T} \leq 12 \mathrm{GeV}$ and $3 \leq p_{T} \leq 12 \mathrm{GeV}$, respectively. In this section, we compare this data with our QMRK predictions. At leading order (LO), we need to consider only three $2 \rightarrow 1$ partonic subprocesses, namely $C_{p} \gamma \rightarrow c$ for direct photoproduction and $C_{p} R_{\gamma} \rightarrow c$ and $R_{p} C_{\gamma} \rightarrow c$ for resolved photoproduction, where the subscripts indicate the mother particles. The corresponding Feynman diagrams are depicted in Figs. 1(a)-1(c), respectively.

Exploiting the factorization theorem, the $p_{T}$ distribution of direct photoproduction takes the form

$$
\begin{aligned}
p_{T}^{3} \frac{d \sigma}{d p_{T}}= & 2 \pi \int d y \int d z x_{\gamma} f_{\gamma / e}\left(x_{\gamma}\right) z^{2} D_{c \rightarrow D}\left(z, \mu^{2}\right) \\
& \times \Phi_{c}^{p}\left(x_{1}, t_{1}, \mu^{2}\right) \overline{\left|M\left(C_{p} \gamma \rightarrow c\right)\right|^{2}},
\end{aligned}
$$

where

$$
\begin{aligned}
& x_{1}=\frac{p_{T} e^{y}}{2 z E_{p}}, \quad x_{\gamma}=\frac{p_{T} e^{-y}}{2 z E_{e}}, \\
& t_{1}=k_{T}^{2}, \quad \vec{k}_{T}=\frac{\vec{p}_{T}}{z},
\end{aligned}
$$

with $\vec{k}_{T}$ being the transverse momentum of the produced charm quark. We evaluate the quasi-real-photon flux $f_{\gamma / e}$ in Weizsäcker-Williams approximation using

$$
\begin{aligned}
f\left(x_{\gamma}\right)= & \frac{\alpha}{2 \pi}\left[\frac{1+\left(1-x_{\gamma}\right)^{2}}{x_{\gamma}} \ln \frac{Q_{\max }^{2}}{Q_{\min }^{2}}\right. \\
& \left.+2 m_{e}^{2} x_{\gamma}\left(\frac{1}{Q_{\min }^{2}}-\frac{1}{Q_{\max }^{2}}\right)\right],
\end{aligned}
$$

where $\alpha$ is Sommerfeld's fine-structure constant, $m_{e}$ is the electron mass, $Q_{\min }^{2}=m_{e}^{2} x_{\gamma}^{2} /\left(1-x_{\gamma}\right)$, and $Q_{\max }^{2}$ is determined by the experimental setup, with $Q_{\max }^{2}=1 \mathrm{GeV}^{2}$ in our case [11,12]. As for the $c \rightarrow D$ fragmentation function (FF) $D_{c \rightarrow D}$, we adopt the nonperturbative $D^{* \pm}$ and $D_{s}^{ \pm}$sets determined in the ZM-VFNS with initial evolution scale $\mu_{0}=m_{c}$ [33] from fits to OPAL data from CERN LEP1. We choose the renormalization and initial- and final-state factorization scales to be $\mu=\sqrt{m_{D}^{2}+p_{T}^{2}}$, where $m_{D}$ is the $D$-meson mass. Using the Reggeized-quark-photon effective vertex from Ref. [6], the square of the hard-scattering amplitude is found to be

$$
\overline{|M(C \gamma \rightarrow c)|^{2}}=4 \pi \alpha e_{c}^{2} k_{T}^{2} .
$$

It is understood that also the contribution from the chargeconjugate partonic subprocess is to be included in Eq. (3).

In the case of resolved photoproduction via the partonic subprocess $C_{p} R_{\gamma} \rightarrow c$, the factorization formula reads:

$$
\begin{aligned}
p_{T}^{3} \frac{d \sigma}{d p_{T}}= & \int d y \int d z \int d x_{\gamma} \int d t_{2} \int d \phi_{2} f_{\gamma / e}\left(x_{\gamma}\right) z^{2} \\
& \times D_{c \rightarrow D}\left(z, \mu^{2}\right) \Phi_{c}^{p}\left(x_{1}, t_{1}, \mu^{2}\right) \Phi_{g}^{\gamma}\left(x_{2}, t_{2}, \mu^{2}\right) \\
& \times \overline{\left|M\left(C_{p} R_{\gamma} \rightarrow c\right)\right|^{2}},
\end{aligned}
$$

where

$$
\begin{aligned}
& x_{1}=\frac{p_{T} e^{y}}{2 z E_{p}}, \quad x_{2}=\frac{p_{T} e^{-y}}{2 x_{\gamma} z E_{e}}, \\
& t_{1}=t_{2}-2 k_{T} \sqrt{t_{2}} \cos \phi_{2}+k_{T}^{2}, \\
& t_{2}=q_{2 T}^{2}, \quad \vec{k}_{T}=\frac{\vec{p}_{T}}{z},
\end{aligned}
$$

with $\phi_{2}$ being the angle enclosed between $\vec{p}_{T}$ and $\vec{q}_{2 T}$. Using the Reggeized-quark-Reggeized-gluon effective vertex from Ref. [24], we have

$$
\overline{|M(C R \rightarrow c)|^{2}}=\frac{2}{3} \pi \alpha_{s}\left(\mu^{2}\right) k_{T}^{2} .
$$

We note that Eq. (6) may be obtained from Eq. (9) by adjusting couplings and color factors. Again, the chargeconjugate partonic subprocess is to be included in Eq. (7). 

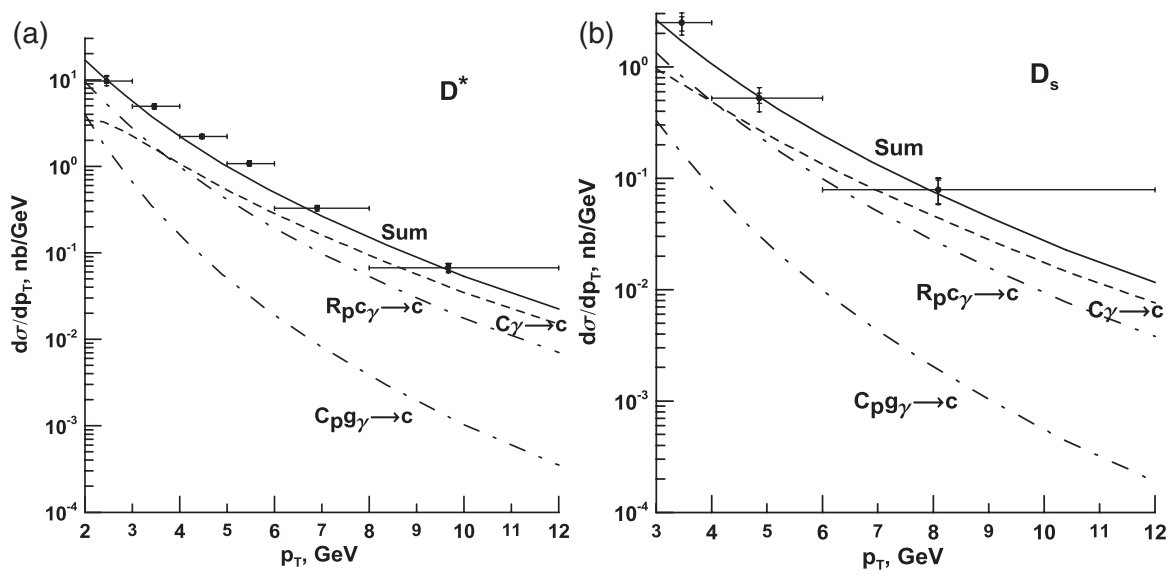

FIG. 3. $p_{T}$ distributions of inclusive (a) $D^{* \pm}$ and (b) $D_{s}^{ \pm}$photoproduction for $\sqrt{S}=300 \mathrm{GeV}$ and $|y| \leq 1.5$. The ZEUS data from (a) Ref. [11] and (b) Ref. [12] are compared with LO predictions from the QMRK approach with the quark-Reggeization hypothesis.

Resolved photoproduction via the partonic subprocess $R_{p} C_{\gamma} \rightarrow c$ is treated very similarly.

Note that Eq. (7) and the corresponding formula for the partonic subprocess $R_{p} C_{\gamma} \rightarrow c$ are only valid in the highenergy limit. Detailed inspection reveals that, under the kinematic conditions of HERA, the average value of $x_{2}$ in these formulas typically exceeds 0.1 . On the other hand, the normalization condition [25]

$$
x F_{a}^{\gamma}\left(x, \mu^{2}\right)=\int_{0}^{\mu^{2}} d q_{T}^{2} \Phi_{a}^{\gamma}\left(x, q_{T}^{2}, \mu^{2}\right),
$$

where $F_{a}$ is the conventional (integrated) PDF of parton $a=g, c$, then breaks down for $x \gtrsim 0.1$. To be on the conservative side, we thus evaluate the resolved-photon contributions to be compared with the ZEUS data $[11,12]$ using conventional photon PDFs, namely, those from Ref. [28].

In Figs. 3(a) and 3(b), our results for $D^{* \pm}$ and $D_{s}^{ \pm}$ mesons, respectively, are broken down to the $C_{p} \gamma \rightarrow c$, $C_{p} g_{\gamma} \rightarrow c$, and $R_{p} c_{\gamma} \rightarrow c$ contributions and are compared with the ZEUS data $[11,12]$. We find that the theoretical predictions are dominated by direct photoproduction and agree rather well with the experimental data over the whole $p_{T}$ range considered.

\section{D-MESON PRODUCTION AT THE TEVATRON}

CDF [7] measured the $p_{T}$ distributions of $D^{0}, D^{ \pm}, D^{* \pm}$, and $D_{s}^{ \pm}$mesons with rapidity $|y| \leq 1$ inclusively produced in hadroproduction in run II at the Tevatron, with $\sqrt{S}=$ $1.96 \mathrm{TeV}$. To LO in the QMRK approach, the factorization formula for the $C_{p} R_{\bar{p}} \rightarrow c$ channel, depicted in Fig. 1(d), reads:

$$
\begin{aligned}
p_{T}^{3} \frac{d \sigma}{d p_{T}}= & \int d y \int d z \int d t_{1} \int d \phi_{1} z^{2} D_{c \rightarrow D}\left(z, \mu^{2}\right) \\
& \times \Phi_{c}^{p}\left(x_{1}, t_{1}, \mu^{2}\right) \Phi_{g}^{\bar{p}}\left(x_{2}, t_{2}, \mu^{2}\right) \overline{\left|M\left(C_{p} R_{\bar{p}} \rightarrow c\right)\right|^{2}},
\end{aligned}
$$

where $\overline{\left|M\left(C_{p} R_{\bar{p}} \rightarrow c\right)\right|^{2}}$ is given by Eq. (9),

$$
\begin{aligned}
& x_{1}=\frac{p_{T} e^{y}}{z \sqrt{S}}, \quad x_{2}=\frac{p_{T} e^{-y}}{z \sqrt{S}}, \\
& t_{2}=t_{1}-2 \frac{p_{T}}{z} \sqrt{t_{1}} \cos \phi_{2}+\frac{p_{T}^{2}}{z^{2}} .
\end{aligned}
$$

The result for the $R_{p} C_{\bar{p}} \rightarrow c$ channel is similar and has to be included in Eq. (11) together with those from the charge-conjugate partonic subprocesses.

In Figs. 4(a)-4(d), our results for $D^{0}, D^{ \pm}, D^{* \pm}$, and $D_{s}^{ \pm}$ mesons, respectively, are compared with the CDF data [7]. We find that the theoretical predictions generally agree rather well with the experimental data, except perhaps for the slope. In fact, the predictions exhibit a slight tendency to undershoot the data at small values of $p_{T}$ and to overshoot them at large values of $p_{T}$. However, we have to bear in mind that these are just LO predictions, so there is room for improvement by including higher orders.

In the framework of the collinear parton model, comparisons with the experimental data of Ref. [7] were performed beyond LO, namely, in the fixed-order-next-toleading-logarithm (FONLL) scheme [8] and at NLO in the GM-VFNS $[9,34]$. The FONLL predictions systematically undershoot the CDF data [7]. The GM-VFNS predictions of Ref. [9], which are evaluated with FFs determined in the ZM-VFNS [33], describe these data within their errors, but are still somewhat on the low side. The degree of agreement is further improved [34] by evaluating the GM-VFNS predictions of Ref. [9] using FFs extracted [35] from a global fit to $B$ - and $Z$-factory data of $e^{+} e^{-}$annihilation in the very same scheme.

\section{CONCLUSIONS}

In this paper, we explored the usefulness of the quarkReggeization hypothesis in the framework of the QMRK approach by studying several observables of inclusive 

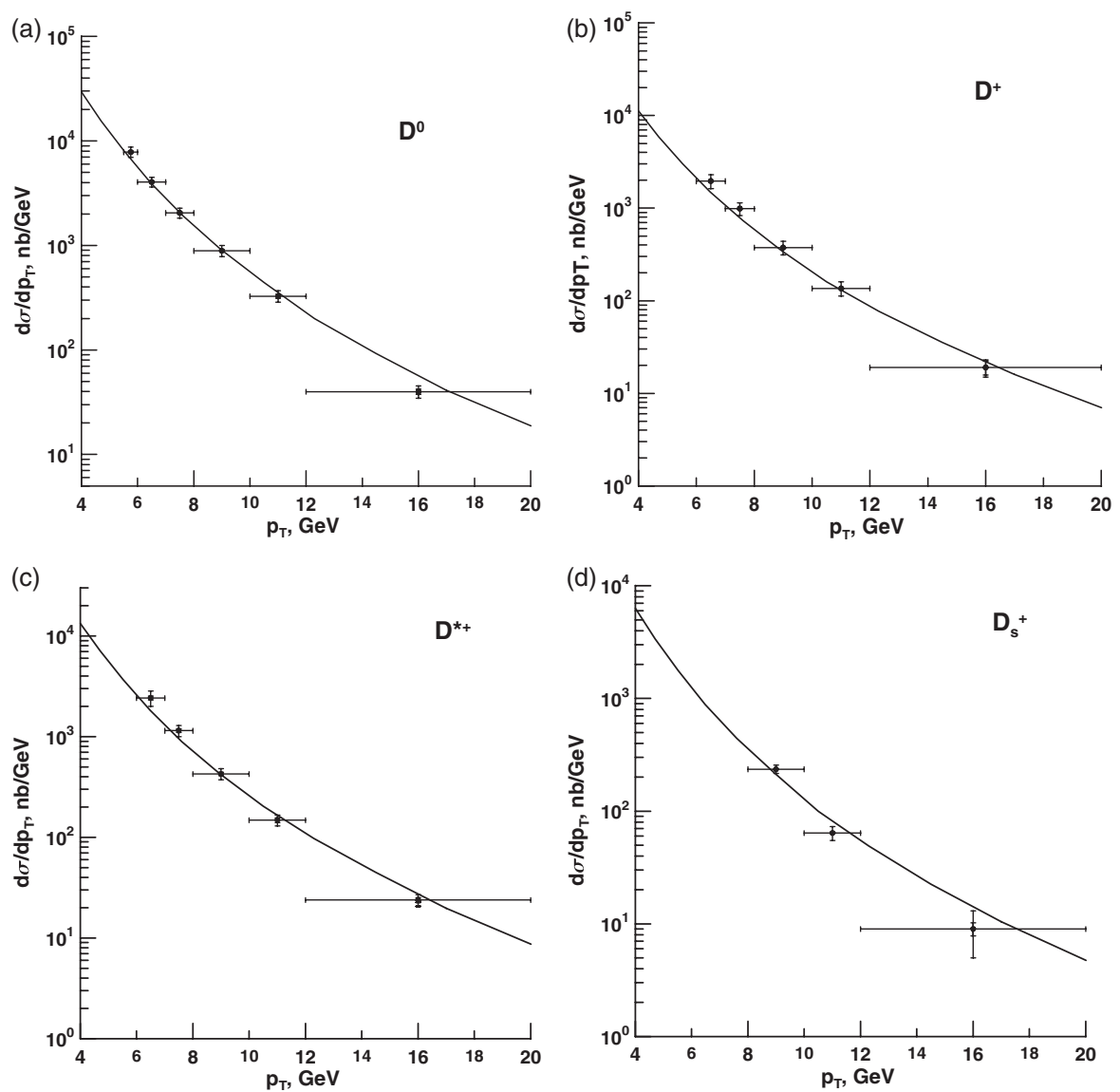

FIG. 4. $p_{T}$ distributions of inclusive (a) $D^{0}$, (b) $D^{ \pm}$, (c) $D^{* \pm}$, and (d) $D_{s}^{ \pm}$hadroproduction for $\sqrt{S}=1.96 \mathrm{TeV}$ and $|y| \leq 1$. The CDF data from Ref. [7] are compared with LO predictions from the QMRK approach with the quark-Reggeization hypothesis.

charm production at LO, namely, the charm structure function $F_{2, c}$ of the proton measured at HERA [29,30] as well as the one-particle-inclusive cross sections of $D^{* \pm}$ and $D_{s}^{ \pm}$photoproduction in $e p$ collisions at HERA $[11,12]$ and of $D^{0}, D^{ \pm}, D^{* \pm}$, and $D_{s}^{ \pm}$hadroproduction in $p \bar{p}$ collisions at the Tevatron [7]. In all three cases, we found satisfactory agreement between our default predictions and the experimental data, which is quite encouraging in view of the simplicity of our LO expressions for the partonic cross sections. By contrast, in the collinear parton model of $\mathrm{QCD}$, the inclusion of NLO corrections is necessary to achieve such a degree of agreement. We thus recover the notion that the QMRK approach is a powerful tool for the theoretical description of QCD processes in the highenergy limit and automatically accommodates an important class of corrections that lie beyond the reach of the collinear parton model at LO [4].

\section{ACKNOWLEDGMENTS}

We thank L. N. Lipatov and O.V. Teryaev for useful discussions. The work of B. A. K. was supported in part by the German Federal Ministry for Education and Research BMBF through Grant No. 05 HT6GUA and by the German Research Foundation DFG through Grant No. KN 365/7-1. The work of A. V. S. was supported in part by Michail Lomonosov Grant No. A/07/97669, jointly funded by the German Academic Exchange Service DAAD and the Russian Ministry of Education and Science, by the International Center of Fundamental Physics in Moscow, and by the Dynastiya Foundation. The work of V.A.S. was supported in part by the Ministry of Education and Science of the Russian Federation through the Analytical Departmental Target Program No. 3341 Development of Scientific Potential of the Higher School. 
[1] V. S. Fadin and L. N. Lipatov, Nucl. Phys. B406, 259 (1993).

[2] V.S. Fadin and L. N. Lipatov, Nucl. Phys. B477, 767 (1996).

[3] L. N. Lipatov, Nucl. Phys. B452, 369 (1995).

[4] V. A. Saleev and D. V. Vasin, Phys. Rev. D 68, 114013 (2003); Yad. Fiz. 68, 95 (2005) [Phys. At. Nucl. 68, 94 (2005)]; Phys. Part. Nucl. 38, 635 (2007); B. A. Kniehl, D. V. Vasin, and V. A. Saleev, Phys. Rev. D 73, 074022 (2006); B. A. Kniehl, V. A. Saleev, and D. V. Vasin, ibid. 74, 014024 (2006).

[5] Ph. Hägler, R. Kirschner, A. Schäfer, L. Szymanowski, and O. V. Teryaev, Phys. Rev. D 62, 071502(R) (2000).

[6] V. A. Saleev, Phys. Rev. D 78, 034033 (2008).

[7] D. E. Acosta et al. (CDF Collaboration), Phys. Rev. Lett. 91, 241804 (2003).

[8] M. Cacciari and P. Nason, J. High Energy Phys. 09 (2003) 006.

[9] B. A. Kniehl, G. Kramer, I. Schienbein, and H. Spiesberger, Phys. Rev. D 71, 014018 (2005); Eur. Phys. J. C 41, 199 (2005); Phys. Rev. Lett. 96, 012001 (2006); Phys. Rev. D 77, 014011 (2008).

[10] S. Aid et al. (H1 Collaboration), Nucl. Phys. B472, 32 (1996); C. Adloff et al. (H1 Collaboration), ibid. B545, 21 (1999).

[11] J. Breitweg et al. (ZEUS Collaboration), Eur. Phys. J. C 6, 67 (1999).

[12] J. Breitweg et al. (ZEUS Collaboration), Phys. Lett. B 481, 213 (2000).

[13] J. Binnewies, B. A. Kniehl, and G. Kramer, Z. Phys. C 76, 677 (1997); B. A. Kniehl, G. Kramer, and M. Spira, ibid. 76, 689 (1997); J. Binnewies, B.A. Kniehl, and G. Kramer, Phys. Rev. D 58, 014014 (1998).

[14] M. Cacciari, M. Greco, S. Rolli, and A. Tanzini, Phys. Rev. D 55, 2736 (1997); M. Cacciari and M. Greco, ibid. 55, 7134 (1997).

[15] S. Frixione, M. L. Mangano, P. Nason, and G. Ridolfi, Phys. Lett. B 348, 633 (1995); S. Frixione, P. Nason, and G. Ridolfi, Nucl. Phys. B454, 3 (1995).

[16] G. Kramer and H. Spiesberger, Eur. Phys. J. C 22, 289 (2001); 28, 495 (2003); 38, 309 (2004); B. A. Kniehl, G. Kramer, I. Schienbein, and H. Spiesberger, Report No. DESY 08-204.

[17] A. V. Berezhnoy, V. V. Kiselev, and A. K. Likhoded, Yad. Fiz. 63, 1682 (2000) [Phys. At. Nucl. 63, 1595 (2000)].

[18] E. A. Kuraev, L. N. Lipatov, and V. S. Fadin, Zh. Eksp. Teor. Fiz. 71, 840 (1976) [Sov. Phys. JETP 44, 443 (1976)]; I. I. Balitsky and L. N. Lipatov, Yad. Fiz. 28, 1597 (1978) [Sov. J. Nucl. Phys. 28, 822 (1978)].

[19] L. V. Gribov, E. M. Levin, and M. G. Ryskin, Phys. Rep. 100, 1 (1983); J. C. Collins and R. K. Ellis, Nucl. Phys. B360, 3 (1991); S. Catani, M. Ciafaloni, and F. Hautmann, ibid. B366, 135 (1991).

[20] V. S. Fadin, M. I. Kotsky, and L. N. Lipatov, Phys. Lett. B 415, 97 (1997); D. Ostrovsky, Phys. Rev. D 62, 054028 (2000); V. S. Fadin, M. G. Kozlov, and A. V. Reznichenko, Yad. Fiz. 67, 377 (2004) [Phys. At. Nucl. 67, 359 (2004)]; J. Bartels, A. Sabio Vera, and F. Schwennsen, J. High Energy Phys. 11 (2006) 051.

[21] E. N. Antonov, L. N. Lipatov, E. A. Kuraev, and I. O. Cherednikov, Nucl. Phys. B721, 111 (2005).

[22] V. S. Fadin and L. N. Lipatov, Pis'ma Zh. Eksp. Teor. Fiz. 49, 311 (1989) [JETP Lett. 49, 352 (1989)]; Yad. Fiz. 50, 1141 (1989) [Sov. J. Nucl. Phys. 50, 712 (1989)].

[23] V. S. Fadin and V. E. Sherman, Pis'ma Zh. Eksp. Teor. Fiz. 23, 599 (1976) [JETP Lett. 23, 548 (1976)]; Zh. Eksp. Teor. Fiz. 72, 1640 (1977) [Sov. Phys. JETP 45, 861 (1977)].

[24] L. N. Lipatov and M. I. Vyazovsky, Nucl. Phys. B597, 399 (2001); A. V. Bogdan and V.S. Fadin, ibid. B740, 36 (2006).

[25] M. A. Kimber, A. D. Martin, and M. G. Ryskin, Phys. Rev. D 63, 114027 (2001); G. Watt, A. D. Martin, and M. G. Ryskin, Eur. Phys. J. C 31, 73 (2003); Phys. Rev. D 70, 014012 (2004); 70, 079902(E) (2004).

[26] G. Watt, http://www.hep.ucl.ac.uk/ watt/.

[27] A. D. Martin, R. G. Roberts, W. J. Stirling, and R. S. Thorne, Phys. Lett. B 531, 216 (2002).

[28] M. Glück, E. Reya, and A. Vogt, Phys. Rev. D 46, 1973 (1992).

[29] C. Adloff et al. (H1 Collaboration), Phys. Lett. B 528, 199 (2002).

[30] S. Chekanov et al. (ZEUS Collaboration), Phys. Rev. D 69, 012004 (2004).

[31] A. Yu. Illarionov, B. A. Kniehl, and A. V. Kotikov, Phys. Lett. B 663, 66 (2008); N. Ya. Ivanov and B. A. Kniehl, Report No. DESY 08-081, arXiv:0806.4705 [Eur. Phys. J. $\mathrm{C}$ (in press)].

[32] Since we neglect finite quark and hadron mass effects, pseudorapidity and rapidity coincide.

[33] J. Binnewies, B. A. Kniehl, and G. Kramer, Phys. Rev. D 58, 014014 (1998); B. A. Kniehl and G. Kramer, ibid. 71, 094013 (2005); 74, 037502 (2006).

[34] B. A. Kniehl, in Proceedings of the XVI International Workshop on Deep-Inelastic Scattering and Related Subjects (DIS 2008), London, England, 2008, edited by R. Devenish and J. Ferrando (Science Wise Publishing, Amsterdam, 2008), http://dx.doi.org/10.3360/ dis.2008.195; B. A. Kniehl, G. Kramer, I. Schienbein, and H. Spiesberger, Report No. DESY 09-008, MZ-TH/ 09-03, LPSC 09-17.

[35] T. Kneesch, B. A. Kniehl, G. Kramer, and I. Schienbein, Nucl. Phys. B799, 34 (2008). 